\title{
Highly Effective Non-Viral Gene Transfer into \\ Vascular Smooth Muscle Cells of Cultured Resistance Arteries Demonstrated by Genetic Inhibition of Sphingosine-1-Phosphate-Induced Vasoconstriction
}

\author{
Steffen-Sebastian Bolz Ulrich Pohl \\ Institute of Physiology, Ludwig Maximilians University, Munich, Germany
}

\section{Key Words}

Hypertension · Plasmids · Rho kinase $\cdot$ RhoA .

Transfection

\begin{abstract}
The linkage of vascular genes to specific functions will lead to a better understanding of cardiovascular pathophysiology. We developed an experimental model that enables the introduction of one or multiple gene(s) into vascular smooth muscle cells (VSMCs) of isolated resistance arteries. Exposure of the arteries to a green fluorescent protein (GFP)-encoding plasmid in combination with the transfectant Effectene ${ }^{\circledR}$ for $20 \mathrm{~h}$ resulted in the expression of GFP in virtually all VSMCs in the arterial wall at fully preserved vascular function. For functional validation of the model, plasmids encoding the specific RhoA inhibitors C3 transferase or N19RhoA were transfected. In subsequent functional tests, inhibition of RhoA-dependent constriction induced by sphingosine-1phosphate was similar to that in arteries treated with exogenous C3 transferase protein or the Rho kinase inhibitor Y27632. Responses to norepinephrine remained unaffected. This novel transfection technique en-
\end{abstract}

ables gene function to be assessed in direct conjunction with signalling pathways in vascular tissue and provides, therefore, a new tool for microvascular proteomics.

Copyright $@ 2003$ S. Karger AG, Basel

\section{Introduction}

Recent studies have shed a new light on how variations in expression patterns of single genes can affect vascular function [1]. To better characterize functional effects of distinct genes and their protein products on the cellular level, overexpression, knockout or targeted mutation of the respective gene are valuable tools. All of these techniques require effective and reliable introduction of barely membrane-permeable DNA into cells in culture or intact tissue. Currently used methods to introduce DNA plasmids into target cells comprise calcium-phosphate precipitation, electroporation, detergent-DNA complexes, microinjections as well as virus- and lipid-mediated transfection. Poorly differentiated tumor-derived cell lines have become the main model for gene expression studies in mammalian cells because in these cells relatively high transfection efficiencies can be achieved. In pri-

\begin{tabular}{ll}
\hline KARGER & @ 2003 S. Karger AG, Basel \\
Fax +41 61 306 1234 $34-1172 / 03 / 0404-0399 \$ 19.50 / 0$ \\
$\begin{array}{l}\text { E-Mail karger@karger.ch } \\
\text { www.karger.com }\end{array}$ & $\begin{array}{l}\text { Accessible online at: } \\
\text { www.karger.com/jvr }\end{array}$
\end{tabular}

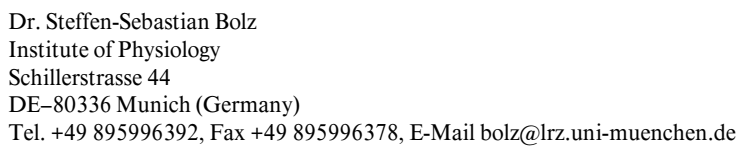


mary cell cultures, however, the uptake of plasmid DNA is usually low and varies considerably between experiments [2], no matter which non-viral transfection method was applied. This holds especially true for vascular smooth muscle cells (VSMC) [3-5] that play a key role in the regulation of vascular tone and in the development of atherosclerosis or hypertension. Recent approaches to transfect VSMCs in culture [6] or intact arterial beds in vivo [7] were primarily based on adenoviral vectors. However, viral strategies bear several disadvantages. They are costly due to their high demands on laboratory equipment and laboratory safety. Furthermore, their use as a tool for basic research, especially under in vivo conditions, is compromised by a variety of undesired and barely controllable effects within the targeted organ or ectopic tissue [7]. Edema formation, for example, and immunologic responses [8] of intact arteries in vivo at the site of virus replication seriously reduce their value as an object for functional studies after explantation.

The aim of the present study was, therefore, to establish a non-viral experimental model that facilitates highly efficient transient transfection of VSMCs in isolated functionally intact resistance arteries that, in contrast to cultured cells, enable direct assessment of vascular functions. Combining a special artery culture protocol [9] with prolonged exposure to an optimized mixture of green fluorescent protein (GFP)-coding plasmid DNA and the nonliposomal lipid transfection reagent Effectene ${ }^{\circledR}$, homogenous expression of GFP throughout the smooth muscle layers of the vessel wall was achieved. Moreover, transfection with plasmids coding for RhoA-inhibiting bacterial C3 transferase or the dominant negative RhoA mutant N19RhoA completely inhibited sphingosine-1-phosphate (S1P)-induced, RhoA-mediated constrictions, indicating functionally relevant gene expression in VSMCs of these resistance arteries.

This new technology, which enables sufficient VSMC transfection and subsequent functional studies, combines advantages of classical approaches in the field of vascular biology and recent advances in transfection technologies and provides a new tool for microvascular proteomics.

\section{Methods}

Drugs

The MOPS-buffered salt solution used in organ bath experiments contained (in mmol/l): $145 \mathrm{NaCl}, 4.7 \mathrm{KCl}, 1.5 \mathrm{CaCl}_{2}, 1.17 \mathrm{MgSO}_{4}$, $1.2 \mathrm{NaH}_{2} \mathrm{PO}_{4}, 2.0$ pyruvate, 0.02 EDTA, 3.0 MOPS and 5.0 glucose. L1 5 medium, Cool Calf ${ }^{\circledR}$ serum, norepinephrine (NE) and acetylcholine (ACh) were purchased from Sigma (Deisenhofen, Germany),
S1P from Biomol (Hamburg, Germany), Fura-2AM from Molecular Probes (Oregon, USA) and $\mathrm{MnCl}_{2}$ from Merck (Darmstadt, Germany). Y27632 [((+)-(R)-trans-4-(1-aminoethyl)-N-(4-pyridyl))] was kindly provided by Welfide, Osaka, Japan. The plasmid containing the gene for bacterial C3 transferase [10] was kindly provided by Dr. Richard Treisman, Imperial Cancer Research Fund Laboratories, London, UK. The dominant-negative RhoA mutant N19RhoA [11] was a kind gift from Dr. Alan Hall, University College London, UK. The promoter-free pGL2 luciferase reporter vector was purchased from Promega (Mannheim, Germany). The transfectant Effectene was purchased from Qiagen, Hilden, Germany, Trans-LT was from Mobitec (Göttingen, Germany).

Concentrations given in the text refer to final bath concentrations.

\section{Preparation of Small Resistance Arteries and Resistance Artery}

Culture

The preparation of the arteries, the technique of calcium and diameter measurements [12] as well as the artery culture method have been described in more detail previously [9]. Briefly, segments of small resistance arteries (maximal outer diameter 205-220 $\mu \mathrm{m}$ ) were excised from the gracilis muscle of hamsters and cannulated with glass micropipettes in a culture setup. The segments were perfused with culture medium at a rate calculated to apply a physiological constant wall shear stress within the range between 15 and $20 \mathrm{~N} / \mathrm{m}^{2}$ to the luminal surface of segments at a transmural pressure of $45 \mathrm{~mm} \mathrm{Hg}$.

\section{Transfection of DNA Plasmids into VSMCs of Intact Resistance Arteries}

For selective transfection of VSMCs by adventitial incubation, the standard culture medium in the organ bath was replaced by $4 \mathrm{ml}$ L15 medium containing 15\% heat-inactivated Cool Calf serum (pH 7.4), 20,000 U/1 penicillin and $20 \mathrm{mg} / 1$ streptomycin, $16 \mu \mathrm{l} / \mathrm{ml}$ Effectene and $5 \mu \mathrm{g}$ of the respective DNA plasmid [pEFmyc containing inserts coding GFP (1,045 bp), bacterial C 3 transferase ( $780 \mathrm{bp}$ ) or N19RhoA]. The resistance arteries were incubated at room temperature $\left(28^{\circ} \mathrm{C}\right)$ for $19-21 \mathrm{~h}$, then washed and incubated with the $\mathrm{Ca}^{2+}$ indicator Fura-2.

Exposure to the transfection solution was limited to 19-21 h because pilot experiments revealed optimal preservation of vascular functions for cytomegalovirus (CMV) promoter-driven plasmids for this time frame.

\section{Determination of Transfection Efficiency, Functional Tests and}

Experimental Protocol

At the end of the transfection period, the resistance arteries were washed with MOPS buffer, placed on the stage of an inverted microscope, warmed up to $37^{\circ} \mathrm{C}$, and, after a further incubation period of $2 \mathrm{~h}$ with the calcium dye Fura-2, stimulated with the vasoconstrictor $\mathrm{NE}(0.3 \mu \mathrm{mol} / \mathrm{l})$ and the endothelium-dependent vasodilator $\mathrm{ACh}$ $(1 \mu \mathrm{mol} / 1)$. Simultaneous measurements of VSMC intracellular $\mathrm{Ca}^{2+}$, $\left[\mathrm{Ca}^{2+}\right]_{i}$, and of the diameter during exposure to NE and ACh were performed as previously described [12] to ensure functional intactness of the arteries (data not shown). Only arteries that developed spontaneous tone ( $>5 \%$ with $8-12 \%$ considered to be normal in this type of arteries $[12,13])$ were used to study functional parameters (38 of 40 vessels). Arteries transfected with GFP-containing plasmid ( $\mathrm{n}=$ 8 ) were studied using confocal laser scanning microscopy to determine the presence and distribution of GFP-related fluorescence (ex- 

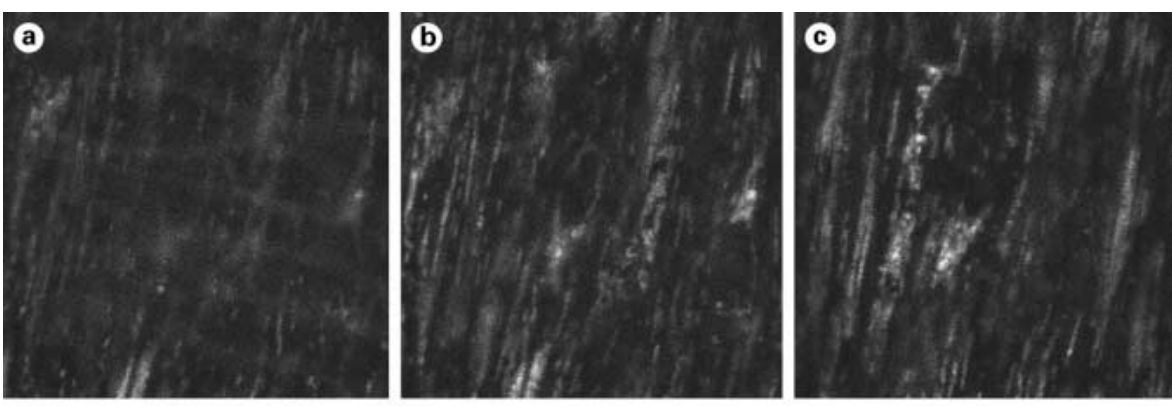

Fig. 1. Serial confocal scans of the microvascular wall. Scans (excitation at $488 \mathrm{~nm}$, emission at $535 \mathrm{~nm}$, pinhole 20) were started at the subendothelial layer of VSMCs (a) and continued stepwise $(1.5 \mu \mathrm{m})$ to the VSMC layer that borders to the adventitial surface of the resistance artery $(\mathbf{b}-\mathbf{f})$. All pictures shown are representative for 5-8 independent experiments.
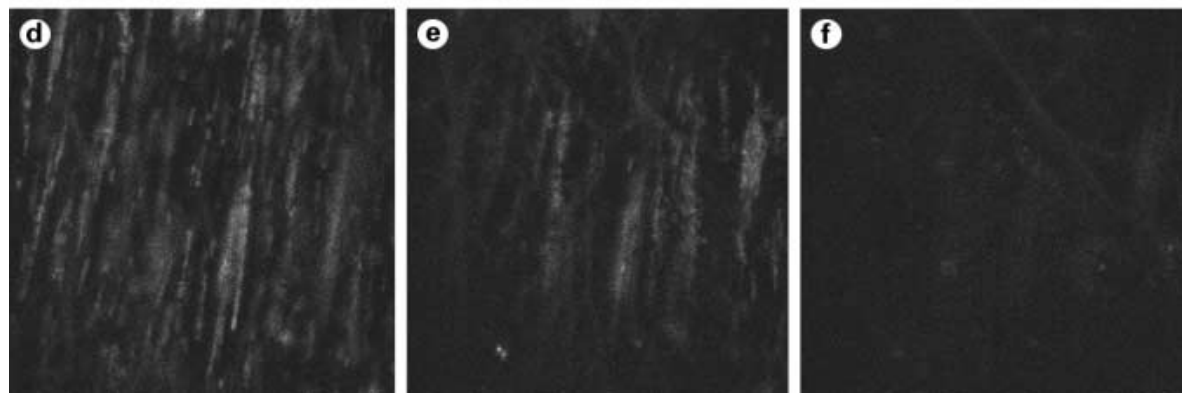

citation at $488 \mathrm{~nm}$, emission at $535 \mathrm{~nm}$ ) within the vascular wall. In order to quantify the transfection efficiency, cell nuclei within the wall of GFP-transfected arteries were selectively stained with propidium iodide (fig. 2g). Subsequently, every stained nucleus was analyzed to determine whether the perinuclear cytosol showed GFPrelated fluorescence. Transfection efficiency (\%) was calculated as $\left.\mathrm{n}_{\text {GFP-related cytosolic fluorescence }} / \mathrm{n}_{\text {stained nuclei }}\right) \cdot 100$. Furthermore, vascular responses of GFP-transfected arteries were compared to those published by us for freshly isolated [12] and for cultured resistance arteries (48 h [9]).

Possible cytotoxic effects of the transfectant Effectene were studied in arteries incubated with this transfectant and a non-replicating plasmid (pGL2).

To study the efficiency of transfections with the C3-transferase or N19RhoA plasmids, transfected arteries were tested for their contractile response to the RhoA activator S1P $(0.003-3 \mu \mathrm{mol} / \mathrm{l})$. The results were compared to those obtained in S1P $(0.003-3 \mu \mathrm{mol} / \mathrm{l})-$ stimulated arteries that were incubated with exogenous bacterial $\mathrm{C} 3$ transferase protein [incubation for $15-17 \mathrm{~h}$ with $\mathrm{L} 15$ culture medium containing antibiotics, the transfectant Trans LT $(20 \mathrm{ml} / \mathrm{ml})$ and C3 transferase protein $(20 \mathrm{mg} / \mathrm{ml}, 16 \mathrm{~h})$ or the Rho kinase inhibitor Y27632 (1 $\mu \mathrm{mol} / 1,10 \mathrm{~min})]$. GFP-overexpressing arteries were used as controls and repeatedly stimulated with S1P (cumulative doseresponse curves, $0.003-3 \mu \mathrm{mol} / \mathrm{l}$ ) to control time- and transfectiondependent unspecific effects.

\section{Statistical Analysis}

Constriction levels after exposure to NE or S1P are expressed as 'percent of maximal diameter' $=\left(\left(\mathrm{dia}_{\max }-\mathrm{dia}_{\mathrm{vc}}\right) / \mathrm{dia}_{\max }\right) \times 100$, with $\mathrm{dia}_{\mathrm{vc}}$ representing the steady state outer diameter $2 \mathrm{~min}$ after stimulation with the respective dose of the vasoconstricting stimulus and $\mathrm{dia}_{\max }$ being the maximal diameter obtained in $\mathrm{Ca}^{2+}$-free MOPS buffer containing $1 \mathrm{mmol} / \mathrm{l}$ EGTA. Measurements were performed at a transmural pressure of $45 \mathrm{~mm} \mathrm{Hg}$.

Transfection of VSMCs in Intact Resistance Arteries
Steady state values from different groups were compared using ANOVA followed by post hoc analysis of the means. Data are presented as means \pm SEM of $n$ experiments, with $n$ representing the number of vessels used per experimental series. Differences were considered significant at an error probability of $p<0.05$.

Curves were compared using a non-linear regression analysis applied first to every individual curve and then to the pooled data. Curves were considered to be different if the F-test indicated a significantly smaller sum of squares for the deviations in each individual fit as compared to the deviation in the fit to the pooled data [14].

\section{Results}

\section{GFP-Related Fluorescence within the Vascular Wall}

As determined by means of confocal microscopy, the vessel wall of third-order skeletal muscle resistance arteries contained three to four layers of VSMCs. The background fluorescence of untransfected arteries upon excitation at $488 \mathrm{~nm}$ was weak and as suggested by figure 1a most likely emitted by matrix components. VSMCs in resistance arteries that were adventitially exposed to incubation solution containing the GFP plasmid for 19-21 h showed GFP-related fluorescence in virtually all VSMCs/ microscopic field (confocal plane) throughout all smooth muscle layers (fig. 1a-f, $2 \mathrm{a}-\mathrm{c}$ ). A quantification of the transfection efficiency was performed in 35 confocal images from five arteries, where $96.5 \pm 1 \%$ of all VSMCs that showed a propidium-iodide-stained nucleus also revealed GFP-related fluorescence within the cytosol. 

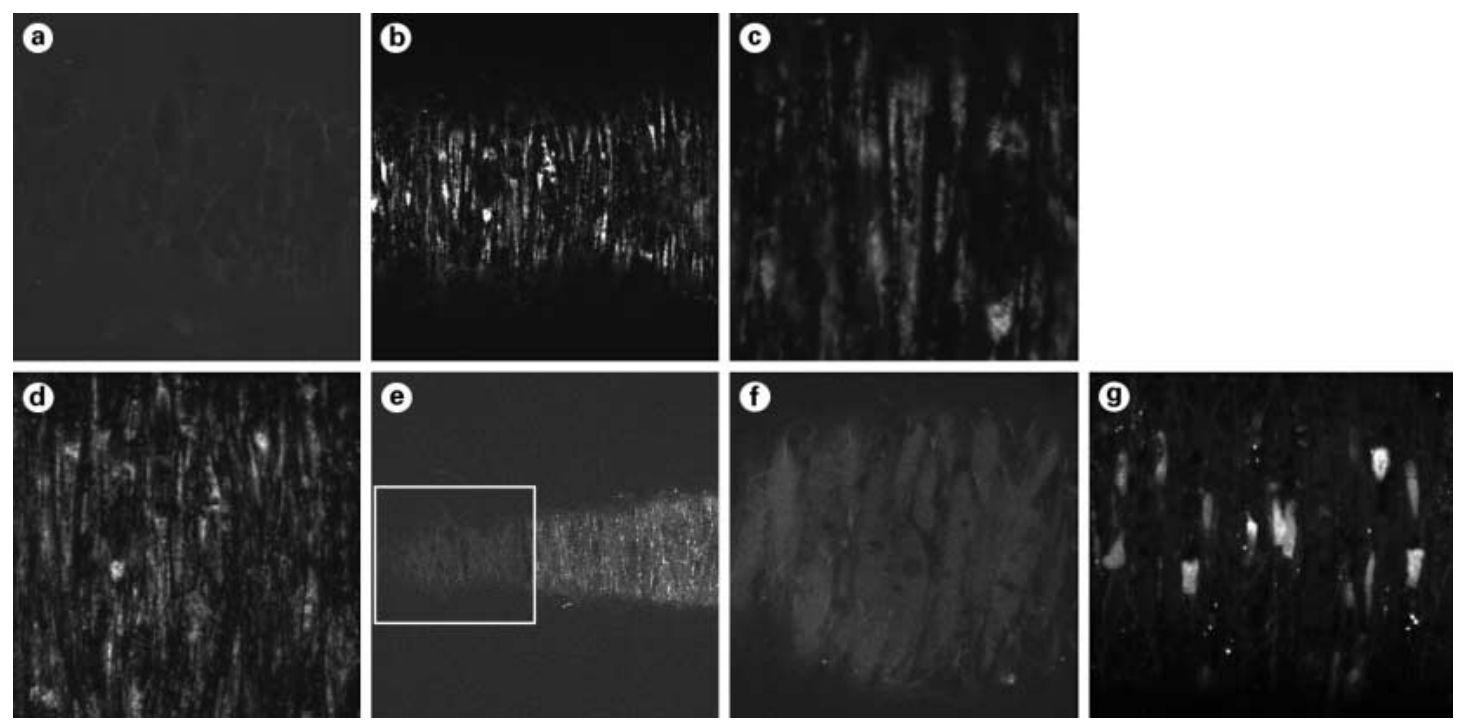

Fig. 2. Expression of GFP in smooth muscle cells of hamster resistance arteries (b-e). Arteries were exposed to a transfection solution containing a plasmid coding for GFP and confocally scanned after transfection periods for 19-21 h. Non-transfected smooth muscle cells were not fluorescent (a), whereas transfected arteries showed homogenous GFP fluorescence confined to the cytosol of smooth muscle cells and absent in the intercellular space. b Overview. $\mathbf{c} \times 3.4$. d A $3 \mathrm{D}$ projection rebuilt from 51 single confocal slices. Continuous excitation of a selected region (dotted square) of the vessel at $488 \mathrm{~nm}$ induced photobleaching (e). f Deformed VSMCs after a 25-hour transfection period. $\mathbf{g}$ Propidium-iodide-stained cell nuclei in GFP-expressing VSMCs. This protocol was used to quantify the transfection efficiency. Pictures are representative for 5-8 independent experiments.

After adventitial incubation, no GFP-related fluorescence was detectable within the endothelium. The fluorescence in VSMCs was strictly intracellular; even at higher magnification no fluorescence was found within the extracellular space (fig. 2d). Continuous excitation at $488 \mathrm{~nm}$ resulted in bleaching of the GFP-related fluorescence (fig. 2e) but did not further reduce the background fluorescence emitted by matrix components, for example. Functional characterization of GFP-transfected arteries revealed intact contractile responses to $0.3 \mu \mathrm{mol} / 1 \mathrm{NE}$ ( 46 $\pm 3 \%, \mathrm{n}=8$, vs. $43 \pm 5 \%$ in freshly isolated and $42 \pm 4 \%$ in cultured resistance arteries [9]) and increases in transmural pressure (from 45 to $110 \mathrm{~mm} \mathrm{Hg}$, Bayliss effect). The latter myogenic responses reversed pressure-induced distensions of GFP-overexpressing resistance arteries by $55 \pm 7 \%(\mathrm{n}=8)$ within $8 \mathrm{~min}$ and were, therefore, not significantly different from those seen in untransfected arteries $(49 \pm 14 \%$ in freshly isolated and $51 \pm 16 \%$ in cultured resistance arteries [9]) that were tested in earlier studies using the same experimental protocol. All arteries transfected with GFP dilated completely upon stimulation with $1 \mu \mathrm{mol} / \mathrm{l}$ of the endothelium-dependent vasodi- lator ACh $(n=8)$, indicating that the transfection did not alter endothelial function.

In vessels that were transfected with the CMV-promoter-driven GFP-containing plasmid for $24 \mathrm{~h}$ or longer, contractile responses to NE, potassium and pressure as well as dilations following ACh were abolished $(n=5)$. Confocal scans at these time points revealed prominent changes in cell shape (fig. 2f).

To exclude cytotoxic effects of the transfectant Effectene, being potentially responsible for the loss of function, arteries were exposed to a mixture of Effectene and a nonreplicating plasmid (pGL2) for 25-27 h. Resting tone (9 $\pm 2 \%$ ), constrictions to $0.3 \mu \mathrm{mol} / 1 \mathrm{NE}(48 \pm 4 \%$ ), myogenic responses $(60 \pm 12 \%)$ and dilations to $1 \mu \mathrm{mol} / 1 \mathrm{ACh}$ $(84 \pm 7 \%)$ in these arteries $(n=4)$ were not different from those in freshly isolated [12], cultured [9] or GFP-transfected (19-21 h) arteries.

\section{S1P-Induced Constrictions in Transfected Arteries}

$\mathrm{S} 1 \mathrm{P}(0.003,0.03,0.3$ and $3 \mu \mathrm{mol})$ induced dose-dependent constrictions of GFP-transfected resistance arteries $(0.2 \pm 0.3,8 \pm 2,27 \pm 3,45 \pm 2 \%, \mathrm{n}=8)$ that were 
completely reversible after washout and not altered after repetitive stimulation. Constrictions in response to 0.003 , 0.03 and $0.3 \mu \mathrm{mol} / 1$ were strictly dependent on RhoA and Rho kinase, since they were abolished by the exogenously applied RhoA-inhibiting bacterial toxin $\mathrm{C} 3$ transferase $(0.7 \pm 0.3,0.5 \pm 0.6,2.8 \pm 0.7 \%, \mathrm{n}=7, \mathrm{p}<0.05$; fig. 3$)$ as well as the Rho kinase inhibitor Y27632 (0.1 $\pm 0.4,0.4$ $\pm 0.3,3.4 \pm 2 \%, \mathrm{n}=7, \mathrm{p}<0.05$, fig. 3). Constrictory responses to $3 \mu \mathrm{mol} / 1 \mathrm{~S} 1 \mathrm{P}$ were significantly reduced (18 \pm 3 and $20 \pm 7 \%$, respectively, each $n=7, p<0.05$ ) but not completely inhibited by either inhibitor.

Genetic inhibition of RhoA in VSMCs with plasmids coding for $\mathrm{C} 3$ transferase or N19RhoA blocked constrictions induced by $0.003,0.03$ and $0.3 \mu \mathrm{mol} / 1 \mathrm{~S} 1 \mathrm{P}$ (C3 transferase plasmid: $1 \pm 0.4,2 \pm 1,4 \pm 2 \%, \mathrm{n}=7$; $\mathrm{p}<$ 0.05; N19RhoA plasmid: $2 \pm 1,3 \pm 2,6 \pm 2 \%, \mathrm{n}=4, \mathrm{p}<$ 0.05 ; both fig. 3).

As shown with the conventional methods to inhibit RhoA/Rho kinase, exogenous $\mathrm{C} 3$ transferase and Y27632, although constrictions in response to $3 \mu \mathrm{mol} / 1$ S1P were significantly reduced (to $21 \pm 5 \%, \mathrm{n}=7, \mathrm{p}<$ 0.05 for the $\mathrm{C} 3$ transferase-containing plasmid and to 18 $\pm 3 \%, \mathrm{n}=4, \mathrm{p}<0.05$ for the N19RhoA plasmid), they were not completely inhibited (fig. 3).

In comparison to GFP-overexpressing arteries, neither transfection with C3 transferase nor with N19RhoA did significantly reduce resting tone (C3 transferase plasmid: $7 \pm 3 \%, \mathrm{n}=7$; N19RhoA plasmid: $6 \pm 3 \%, \mathrm{n}=4$ ) or constrictions induced by $0.3 \mu \mathrm{mol} / 1 \mathrm{NE}$ (C3 transferase plasmid: $48 \pm 5 \%$; N19RhoA plasmid: $52 \pm 4 \%$ ).

\section{Discussion}

This study describes a novel transfection model that enables highly efficient non-viral transfection of VSMC with DNA plasmids in an intact arterial preparation. Functional tests after transfection with a plasmid coding for the heterogenic jellyfish GFP, which should not interfere with the regulation of vascular functions, revealed that the transfection procedure per se did not affect vascular responses to vasoconstricting or dilating stimuli.

In contrast, overexpression of two proteins with known RhoA-inhibiting properties (C3 transferase and N19RhoA) abolished S1P-induced constrictions, suggesting that the latter are mediated by RhoA and that the expression level of the transfected proteins was sufficient to interfere in a highly specific manner with the S1P signalling pathway in VSMCs of these arteries.

Transfection of VSMCs in Intact Resistance Arteries

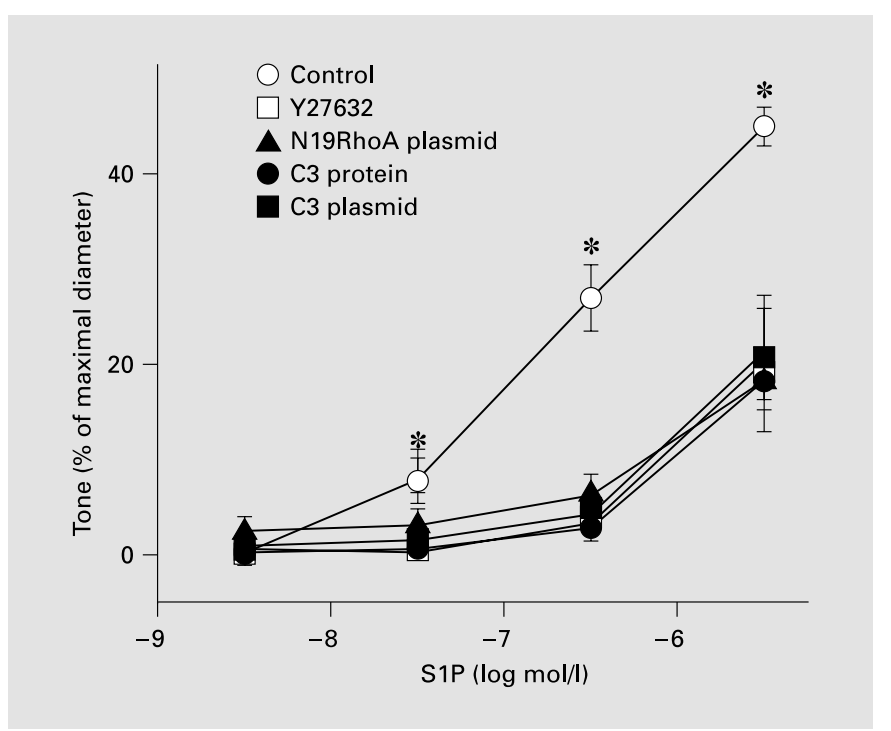

Fig. 3. $\mathrm{S} 1 \mathrm{P}(0.003-0.3 \mu \mathrm{mol} / 1)$-induced, RhoA-mediated constrictions of resistance arteries (control) were abolished after transfection of plasmids coding for bacterial C3 transferase $(n=7)$ or the dominant negative RhoA mutant N19RhoA $(n=4)$. The gene products of these plasmids had virtually identical effects on S1P-induced constriction as exogenous C3 transferase $(n=7)$ or Y27632 $(n=7)$, respectively. Means $\pm \mathrm{SEM} ;{ }^{*} \mathrm{p}<0.05$ vs. $\mathrm{C} 3$ or N19RhoA plasmidtransfected arteries.

This new model is the first to provide a tool for sufficient non-viral genetic manipulation of VSMCs in intact isolated resistance arteries to immediately assess functional changes evoked by the protein product of the transfected gene.

VSMCs in non-transfected arteries emitted only weak background fluorescence upon excitation with $488 \mathrm{~nm}$. Nineteen to $21 \mathrm{~h}$ after transfection with a GFP-coding plasmid, expression of GFP could be determined based on its characteristic fluorescence in virtually all VSMCs of the vascular wall. In contrast to matrix-related autofluorescence, cytosolic fluorescence was sensitive to photobleaching by continuous excitation at $488 \mathrm{~nm}$, further supporting the assumption that the cytosolic fluorescence that is not present in untransfected arteries was related to the alien protein GFP. Not only outer but also subendothelial layers of VSMC of the vascular wall showed GFPrelated fluorescence, indicating sufficient penetration of the plasmid/transfection reagent mixture. However, the basal membrane between VSMC and endothelial cells seems to provide a tight barrier because none of the endothelial cells expressed GFP. This is in agreement with an

J Vasc Res 2003;40:399-405 403 
earlier study from our group where FITC-labeled antisense oligonucleotides against cytochrome $\mathrm{P}_{450} 2 \mathrm{C} 8$ were transfected from the luminal side and remained confined to the endothelium [13].

The small GTPase RhoA in smooth muscle cells of these small arteries was genetically inhibited using plasmids coding for bacterial C3 transferase or for the dominant negative RhoA mutant N19RhoA. The inhibition of S1P-induced and RhoA-mediated constrictions by both constructs was virtually the same as that seen with exogenously administered C3 transferase or Y27632. The inhibition of $\mathrm{S} 1 \mathrm{P}$-induced contractile responses resulting from transcription of C3 transferase- or N19RhoA-encoding plasmids, respectively, was homogenous along the cannulated resistance artery. Since N19RhoA and C3 transferase (molecular weights between 21 and $25 \mathrm{kD}$ ) are too large to cross gap junctions and results from dye transfer experiments suggest that VSMC within the vascular wall of this resistance artery model are barely coupled [Bolz et al., unpubl. data], a homogenous inactivation of RhoA requires expression of $\mathrm{C} 3$ or N19RhoA in all VSMC. Contractile responses to a submaximal dose of the $\alpha$ agonist NE remained unaffected in C3 transferase- or N19RhoA-transfected arteries. This is in accordance with a recent paper [15] demonstrating that the $\alpha$ agonist phenylephrine did not affect the activity of the smooth muscle myosin light chain phosphatase which is the effector protein for the RhoA/Rho kinase pathway in VSMC. In the context of the present study, the unaffected responses to NE in C3 transferase or N19RhoA-overexpressing arteries underline the specificity of the genetic inhibition of RhoA.

The results presented here suggest that genetic constructs coding for either a bacterial inhibitory toxin or an inhibitory mutant of the targeted protein could replace chemical inhibitors for microvascular research. Therefore, the use of genetic inhibitors according to the protocol presented here provides a promising strategy to overcome the lack of specificity of chemical inhibitors that often compromise the results obtained.

In the transfection experiments presented in this study with both plasmids being driven by a CMV promoter, the transfection period was kept between 19 and maximally $21 \mathrm{~h}$ in order to obtain optimal functional results. The reason for the decline in vascular reactivity after transfection periods that exceeded the indicated time frame could be that the transcription of plasmids is driven by strong viral promoters, in this case from CMV. These viral promoters recruit large parts of the protein biosynthesis apparatus of the transfected host cell to produce high amounts of plasmid-encoded alien protein in short time. Due to its viral nature, this promotor cannot be regulated by the host cell. To share limited cellular resources, the permanently necessary regeneration of cellular proteins has to slow down, resulting in a net loss of structural and functional cellular proteins like actin and myosin, and could be the reason for the complete loss of contractility along with cell deformation found in arteries after transfection periods exceeding $24 \mathrm{~h}$. As a consequence, it will be necessary to adjust the transfection protocol to the strength of each particular viral promoter used by 'titrating' the optimal transfection time. The protocol used in this study was optimized for the CMV promoter.

Cytotoxic effects of the transfectant Effectene per se could be ruled out as a cause for the loss of vascular function because even prolonged exposure (up to $27 \mathrm{~h}$ ) to a mixture of Effectene and a non-replicating plasmid did not affect vascular responses.

In summary, this study introduces a novel model for the assessment of functional properties of vascular relevant genes in intact isolated resistance arteries. It provides a new tool to explore the genetic background of microvascular physiology, most likely resulting in the identification and characterization of new targets for innovative therapeutic strategies. 


\section{References}

1 Fairchild TA, Fulton D, Fontana JT, et al: Acidic hydrolysis as a mechanism for the cleavage of the Glu(298)->Asp variant of human endothelial nitric-oxide synthase. J Biol Chem 2001;276:26674-26679.

2 Kaiser S, Toborek M: Liposome-mediated high-efficiency transfection of human endothelial cells. J Vasc Res 2001;38:133-143.

3 Huang QQ, Fisher SA, Brozovich FV: Forced expression of essential myosin light chain isoforms demonstrates their role in smooth muscle force production. J Biol Chem 1999;274: 35095-35098.

4 Maasch C, Wagner S, Lindschau C, et al: Protein kinase $\mathrm{C} \alpha$ targeting is regulated by temporal and spatial changes in intracellular free calcium concentration $[\mathrm{Ca}(2+)](\mathrm{i})$. FASEB J 2000; 14:1653-1663.

5 Seki N, Hashimoto N, Suzuki Y, et al: Role of SRC homology 2-containing tyrosine phosphatase 2 on proliferation of rat smooth muscle cells. Arterioscler Thromb Vasc Biol 2002;22: 1081-1085.
6 Sekine O, Nishio Y, Egawa K, et al: Insulin activates CCAAT/enhancer binding proteins and proinflammatory gene expression through the phosphatidylinositol 3-kinase pathway in vascular smooth muscle cells. J Biol Chem 2002;277:36631-36639.

7 Vajanto I, Rissanen TT, Rutanen J, et al: Evaluation of angiogenesis and side effects in ischemic rabbit hindlimbs after intramuscular injection of adenoviral vectors encoding VEGF and LacZ. J Gene Med 2002;4:371-380.

8 Vassalli G, Agah R, Qiao R, et al: A mouse model of arterial gene transfer: Antigen-specific immunity is a minor determinant of the early loss of adenovirus-mediated transgene expression. Circ Res 1999;85:e25-e32.

9 Bolz SS, Pieperhoff S, de Wit C, et al: Intact endothelial and smooth muscle function in small resistance arteries after $48 \mathrm{~h}$ in vessel culture. Am J Physiol 2000;279:H1434-H1439.

10 Aktories K, Hall A: Botulinum ADP-ribosyltransferase C3: A new tool to study low molecular weight GTP-binding proteins. Trends Pharmacol Sci 1989;10:415-418.
11 Qiu RG, Chen J, McCormick F, et al: A role for Rho in Ras transformation. Proc Natl Acad Sci USA 1995;92:11781-11785.

12 Bolz SS, de Wit C, Pohl U: Endotheliumderived hyperpolarizing factor but not NO reduces smooth muscle $\mathrm{Ca}^{2+}$ during acetylcholine-induced dilation of microvessels. $\mathrm{Br} \mathrm{J}$ Pharmacol 1999;128:124-134.

13 Bolz SS, Fisslthaler B, Pieperhoff S, et al: Antisense oligonucleotides against cytochrome $\mathrm{P}_{450}$ $2 \mathrm{C} 8$ attenuate EDHF-mediated $\mathrm{Ca}(2+)$ changes and dilation in isolated resistance arteries. FASEB J 2000;14:255-260.

14 Motulsky HJ, Ransnas LA: Fitting curves to data using nonlinear regression: A practical and nonmathematical review. FASEB J 1987; 1:365-374.

15 Shin HM, Je HD, Gallant C, et al: Differential association and localization of myosin phosphatase subunits during agonist-induced signal transduction in smooth muscle. Circ Res 2002; 90:546-553. 